\title{
Using Color Spaces HSV, YIQ and Comparison in Analysis Hazy Image Quality
}

\author{
Firas S. Abdulameer \\ Department of Physics, Collage of Sciences, Mustansiriyah University, PO box 46007, Iraq-Baghdad
}

\begin{abstract}
Analysis images hazy is difficult problem, thus these images need to determined quality the after dehazing. In this paper, were compared to, HSV, YIQ color spaces, adopted in analyze the dust images. we designed the system captured images which graded for high to very low dusty by using $\mathrm{HeNe}$ laser, in these images we calculated the normalize mean square error (NMSE) for each components in HSV, YIQ and RGB color space separately, and the basic components in the structure similarity Index (SSIM) are (contrast, structure and luminance) moreover the mean for all has been calculated. We noted from results spaces, HSV, YIQ chromatic that there is a similarity in analysis of images dusty when compared. This means that the use of any color space gives almost the same results
\end{abstract}

Keywords: The hazy image, contrast, Image quality, Intensity, luminance HSV, YIQ and RGB color spaces.

DOI: $10.7176 /$ APTA/76-03

Publication date:March $31^{\text {st }} 2019$

\section{Introduction}

Image quality is a characteristic of an image that measures the Image degradation. In many cases the image of outdoor scenes are degrade by the improper weather Condition. We have also know that light reflected from a surface is scatted in the atmosphere before it reaches the camera is Due to the presence of aerosol such as dust must and fames[1].Dust weather can significantly influence the quality of images since capturing often leads to undesirable degradation such as poor contrast, deficient colors or color cast[2]. According to the visibility of dust weather there are floating, dust blowing, sandstorms and strong sandstorms. Dust will affect scattering and absorption of light, hence it will cause severe degradation of video images [3].dusty image analysis it is important case in the dehazing image and image quality assessment, the problems which affect in quality analysis of image can be divided into two major categories: dynamic and static. Dynamic problem is such problem which appearing when objects fast-move such as motion tremors and motion blur; static problem is that always occur in a video sequence, and have no relation with object movement, such as various of noise, edge blur, poor contrast[3,4]. In this paper, the dusty color images have been analyzed depending on two objectives quality are the SSIM and NMSR. The each component of Chromaticity spaces, HSV, YIQ are employed in SSIM and NMSR and we compared between them for each space, the important of this procedure is determine the quality of dusty image and the style of it enhanced. In this search we attempt to answer the question what is the space more effective in dusty image analysis. Moreover the components of SSIM in the lightness (Luminance, structure and contrast) it has studied.

\section{HSV Color space}

The three components in HSV color model are hue (H), saturation (S) and value (V). Hue is an attribute associated with the dominant wavelength in a mixture of light waves [5]. For example, a light wave with central tendency of 565 to $590 \mathrm{~nm}$ will be perceived as "yellow" by human observer. In HSV color model, hue represents the dominant color as observed by the human eye and measured in degree from $0^{\circ}$ to $360^{\circ}$. Saturation measures how vivid or pure a color is and the purity refers the amount of "white" color mixed with a hue [5]. A highly saturated color implies a pure color while no saturation makes the hue appear grey. The degree of saturation is inversely proportional to the amount of white light added and white color has zero saturation. Value represents brightness of a color. While hue and saturation defines chromaticity, value represents the achromatic notion of its intensity. Pure achromatic colors range from black to white with all the possible gray colors in between. HSV color space can be represented in various ways [5,6,7]. One typical representation is using a hexagonal disk model The saturation $\mathrm{S}$ and hue $\mathrm{H}$ components specify a point inside the hexagonal disk, Saturation for a given level of $\mathrm{V}$ is defined as the relative length of the vector that points to the given color to the length of the vector that points to the corresponding color on the border or the hexagonal disk [6]. This results in a set of loci of constant $\mathrm{S}$ as shown in figure (1). 


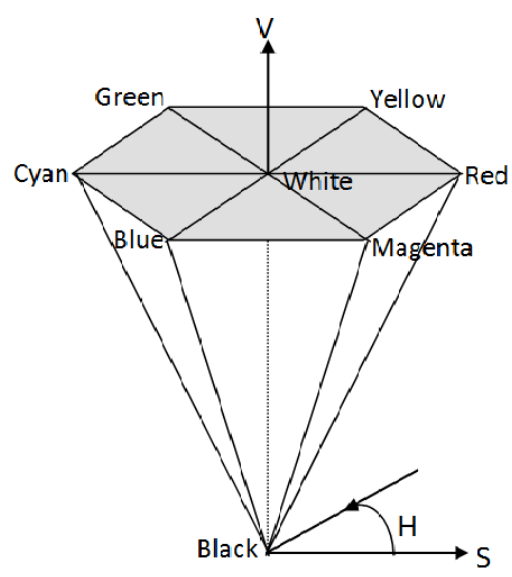

Figure1. The HSV Color space

The transformation from RGB color space to HSV color space is given by [6]:

$$
\begin{gathered}
\mathrm{V}=\max \{\mathrm{R}, \mathrm{G}, \mathrm{B}\} \\
\mathrm{S}=(\max -\min ) / \max \\
H=\left\{\begin{array}{c}
\frac{G-B}{6(\max -\min )} \\
\frac{1}{6}\left(2+\frac{B-R}{\max -\min }\right) \\
\frac{1}{6}\left(4+\frac{R-G}{\max -\min }\right)
\end{array}\right.
\end{gathered}
$$

Where $\max =\max \{\mathrm{R}, \mathrm{G}, \mathrm{B}\}$ and $\min =\min \{\mathrm{R}, \mathrm{G}, \mathrm{B}\}$. All three components $\mathrm{V}, \mathrm{S}$, and $\mathrm{H}$ are in the range $[0,1]$. The transformation from HSV back to RGB is given by [6]:

$$
[\mathrm{R}, \mathrm{G}, \mathrm{B}]=[\mathrm{V}, \mathrm{V}, \mathrm{V}] \text { if } \mathrm{S}=0
$$

If the saturation is not zero, then the RGB components are given:

Where $M, N$, and $K$ are defined as:

$$
[R, G, B]=\left\{\begin{array}{c}
{[V, K, M] \text { if } 0 \leq H<1 / 6} \\
{[N, V, M] \text { if } 1 / 6 \leq H<2 / 6} \\
{[M, V, K] \text { if } 2 / 6 \leq H<3 / 6} \\
{[M, N, V] \text { if } 3 / 6 \leq H<4 / 6} \\
{[K, M, V] \text { if } 4 / 6 \leq H<5 / 6} \\
{[V, M, N] \text { if } 5 / 6 \leq H<1}
\end{array}\right.
$$

And

$$
\begin{gathered}
M=V(1-S) \\
N=V(1-S F) \\
K=V(1-S(1-F))
\end{gathered}
$$

$$
F=6 H-V
$$

\section{YIQ Color space}

In the development of the NTSC television system used in the United States, a color coordinate system with the coordinates Y, I, and Q is defined for transmission purposes. To transmit a color signal efficiently, the R,G, and $\mathrm{B}$ signal is more conveniently coded from a linear transformation. The luminance Signal is coded in the $\mathrm{Y}$ component. The additional portions I and Q contain the entire chromaticity information that is also denoted as chrominance signal in television technology [8]. (I) component containing orange-cyan hue information,and (Q) containing green-magenta hue information. Transform color image from basic RGB color space to YIQ color space by preformed by [6]: And the MSRCR had been applied on eq. (10)[3].

$$
M_{R G B \text { to YIQ }}=\left[\begin{array}{ccc}
0.299 & 0.587 & 0.114 \\
0.596 & -0.270 & -0.322 \\
0.211 & -0.253 & 0.312
\end{array}\right]
$$


While the inverse transformation is given by:

$$
M_{\text {YIQ to RGB }}=\left[\begin{array}{ccr}
1 & 0.956 & 0.621 \\
1 & -0.272 & -0.647 \\
1 & -1.060 & 1.703
\end{array}\right]
$$

There are two peculiarities with the YIQ color model, the first is that this system is more sensitive to changes in luminance than to changes in chromaticity; the second is that color gamut is quite small; it can be specified adequately with one rather than two color dimensions. These properties are very convenient for the transfer of TV signals [8].

\section{Image Quality Assessment by Distortion Model}

Images may be corrupted by degradation such as linear frequency distortion, noise, dust and blocking artifacts. These sources of degradation may arise during image capture or processing, and have a direct bearing on visual quality [9]. In image processing, the model widely used to describe the formation of a haze image is given by $[10,11]$ :

$$
\mathrm{I}(\mathrm{x})=\mathrm{J}(\mathrm{x}) \mathrm{t}(\mathrm{x})+\mathrm{A}(1-\mathrm{t}(\mathrm{x})) \quad(12)
$$

A is the global atmospheric light, and $t$ is medium transmission $\mathrm{J}(\mathrm{x})$ is the original surface radiance vector at the intersection point of the scene with the real-world ray. $J(x) t(x)$ is direct attenuation term [11], representing the scene radiation decay effect in the medium , $\mathrm{A}(1-\mathrm{t}(\mathrm{x}))$ is air light term [11], describing the light scattering from atmosphere particles inducing color distortion. The SSIM metric is based on the evaluation of three different measures, the luminance, contrast, and structure comparison measures are computed as [12]:

$$
\begin{gathered}
l(x, y)=\frac{2 \mu_{X}(x, y) \mu_{Y}(x, y)+C_{1}}{\mu_{X}^{2}(x, y)+\mu_{Y}^{2}(x, y)+C_{1}} \\
c(x, y)=\frac{2 \sigma_{X}(x, y) \sigma_{Y}(x, y)+C_{2}}{\sigma_{X}^{2}(x, y)+\sigma_{Y}^{2}(x, y)+C_{2}} \\
s(x, y)=\frac{\sigma_{X Y}(x, y)+C_{3}}{\sigma_{X}(x, y) \sigma_{Y}(x, y)+C_{3}}
\end{gathered}
$$

Where $\mathrm{X}$ and $\mathrm{Y}$ correspond to two different images that we would like to match, i.e. two different blocks in two separate images, , and the mean of $\mathrm{X}$, the variance of $\mathrm{X}$, and the covariance of $\mathrm{X}$ and $\mathrm{Y}$ respectively where[12]:

$$
\begin{gathered}
\mu(x, y)=\sum_{p=-P}^{P} \sum_{q=-Q}^{Q} w(p, q) X(x+p, y+q) \ldots(13) \sigma^{2}(\mathrm{x}, \mathrm{y}) \\
=\sum_{\mathrm{p}=-\mathrm{P}} \sum_{\mathrm{q}=-\mathrm{Q}}^{\mathrm{Q}} \mathrm{w}(\mathrm{p}, \mathrm{q})\left[\mathrm{X}(\mathrm{x}+\mathrm{p}, \mathrm{y}+\mathrm{q})-\mu_{\mathrm{X}}(\mathrm{x}, \mathrm{y})\right]^{2} \\
\sigma_{X Y}(x, y)=\sum_{p=-P}^{P} \sum_{q=-Q}^{Q} w(p, q)\left[X(x+p, y+q)-\mu_{X}(x, y)\right] \\
{\left[\mathrm{Y}(\mathrm{x}+\mathrm{p}, \mathrm{y}+\mathrm{q})-\mu_{\mathrm{Y}}(\mathrm{x}, \mathrm{y})\right]}
\end{gathered}
$$

Where $w(p, q)$ is a Gaussian weighing function such that:

$$
\sum_{\mathrm{p}=-\mathrm{P}}^{\mathrm{P}} \sum_{\mathrm{q}=-\mathrm{Q}}^{\mathrm{Q}} \mathrm{w}(\mathrm{p}, \mathrm{q})=1
$$

And $C_{1}, C_{2}$, and $C_{3}$ are constants given by $C_{1}=\left(K_{1} L\right)^{2}, C_{2}=\left(K_{2} L\right)^{2}$, and $C_{3}=C_{2} / 2$. $\mathrm{L}$ is the dynamic range for the sample data, i.e. $L=255$ for 8 bit content and $K_{1}<<1$ and $K_{2}<<1$ are two scalar constants. Given the above measures the structural similarity can be computed as [12]:

$$
\operatorname{SSIM}(x, y)=[l(x, y)] \cdot[c(x, y)] \cdot[s(x, y)]
$$

\section{Results and discussion}

A system has been designed to measure the amount of the particle of dust that is distorted the image, which is made up of firm glass box, Fan, laser HeNe, Camera stand, camera and Lux meter as in Figure (2). The Fan is used to stirs the dust that is putting in the box in the same time the lux meter measured the laser intensity. When a specific amount of the dust is added in the box the fun will be stir the dust, after small time the dust is gets still at the bottom of the box. The images are taken from this time during the dust is stirs to it gets on the bottom, we 
can consider all images are the dust image but the last image is approximately optimal images.in this system if the dust is increased the laser intensity is decreased. Figures (3) and (4) the images used in the study which shows the amount of dust from the high to low. In the figures (5a,7a,9a,11a) illustrated the relationships between the max.illuminance and the SSIM for the value component in YIQ, HSV color spaces for data images, from these figures we can see the max.illuminance increasing with increased the SSIM due to decrease the dust. And the relationships between the max.illuminance and SSIM components was illustrate in the figures (5b $, 7 \mathrm{~b}, 9 \mathrm{~b}, 11 \mathrm{~b}$ ) to spaces chromatic (YIQ,HSV), we noted the contrast component is approximately remaining constant whereas the behaviors of structure and luminance are similar the value component, but the SSIM is high in the contrast. The figures $(6 \mathrm{ab}, 8 \mathrm{ab}, 10 \mathrm{ab}, 12 \mathrm{ab})$ are illustrated the relationship between the max.illuminance and the NMSE for RGB and each component in YIQ, HSV for data images, we saw the NMSE is decreasing with increased the max.illuminance in the all component, and the chromatic components I ,Q, hue and saturation are more effected by the dust due to the error is high, because increasing the white light in these images.



Figure2.Rrepresents the system used to measure the amount of dust on the scene

\section{Conclusion}

In this paper, were compared to HSV, YIQ color spaces adopted in study and analyze the dust images, and it gave almost the same results in the analysis of images of dust, since the metric SSIM for value the structure follows the contrast is decreasing due to the dust compare with the luminance in both color spaces. It means that the use of any color space gives almost the same results.

\section{References}

1- Soni Bhamberl, Nilesh Shinde,(2014) Removing Haze, Dust, Aerosol from Image \& Authentication, , International Journal of Advances in Engineering and Applied Science (IJAEAS) Vol-1 Iss-2.

2- Zohair Al-Ameen, (2016).Visibility Enhancement for Images Captured in Dusty Weather via Tuned Trithreshold Fuzzy Intensification Operators, I.J. Intelligent Systems and Applications, DOI: .5815/ijisa,2.08.

3- H. Madokoro, K. Sato, (2013),Hardware implementation of back-propagation neural networks for real-time video image learning and processing, Journal of Computers. vol. 8, pp. 559-566, March 4- Z. 4- L. Zhang, (2010).Research on video enhancement algorithm in video post-processing Beijing University Of Posts And Telecommunications.Beijing,

5- Haim Levkowitz, (1997).Color theory and modeling for computer graphics, visualization, and multimedia applications, Kluwer Academic Publishers,.

6- Marc Ebner,(2007). Color Constancy, John Wiley \& Sons.

7- Sony Wine S., J and Horne R.E., (1998).The Color Image Processing Hand Book, International 8-Thomson, Haim Levkowitz,,(1997), Color theory and modeling for computer graphics, visualization, and multimedia applications ", Kluwer Academic Publishers,.

9- T. D. Kite, B. L. Evans, A. C. Bovik, and T. L. Sculley, (Oct. 1997). Digital halftoning as 2-D delta-sigma modulation, in Proc. IEEE Int. Conf. Image Proc., vol. 1, , pp. 799-802.

10- Kaiming He, Jian Sun,Xiaoou Tang,(2011). Single Image Haze Removal Using Dark Channel Prior, Pattern Analysis and Machine Intelligence, IEEE, vol 33: issue 12 , p 2341 - 2353 . Dec.. 
11- Johannes Kopf, Boris Neubert, Billy Chen, Michael Cohen, Daniel Cohen-Or, Oliver Deussen, Matt Uyttendaele, andDani Lischinski. Deep photo,(2008): Model-based photograph enhancement and viewing. ACM Transactions on Graphics(Proceedings of SIGGRAPH Asia).

12- W.S. Malpica, A.C. Bovik, ,(2009).SSIM based range image quality assessment, Fourth International workshop on Video Processing and Quality Metrics for Consumer Electronics Scottsdale Arizon,.

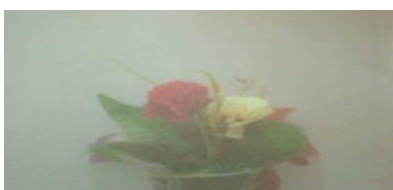

$\mathrm{X} 1$

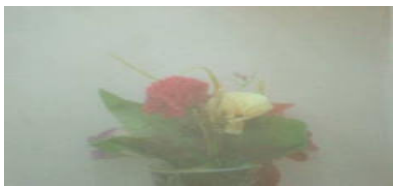

$\mathrm{X} 2$

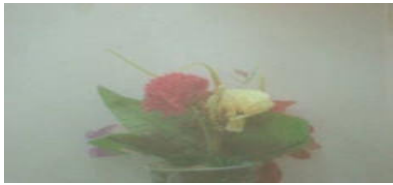

$\mathrm{X} 3$

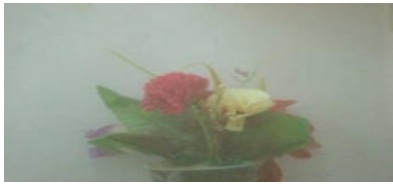

$\mathrm{X} 4$

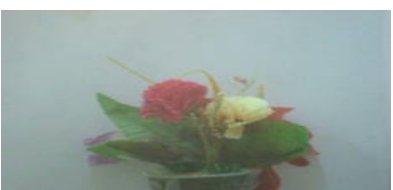

X5



X6

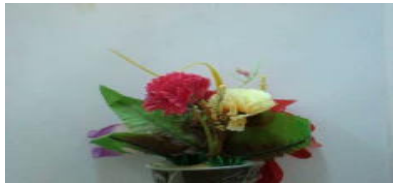

$\mathrm{X} 7$

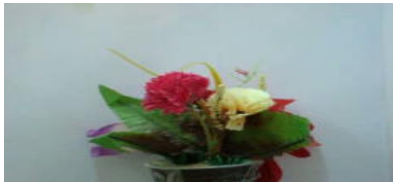

$\mathrm{X} 8$

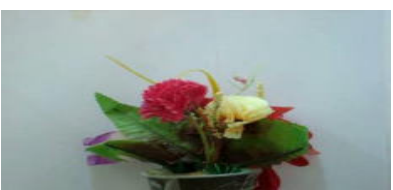

X9

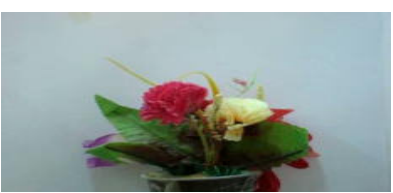

$\mathrm{X} 10$



$\mathrm{X} 11$



$\mathrm{X} 12$

Figure3. First group of the dust images with different dust levels from maximum in $\mathrm{x} 1$ to very low in $\mathrm{x} 12$ 


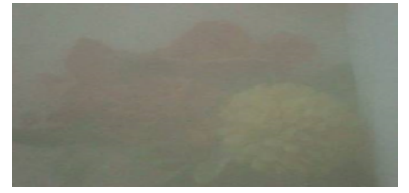

$\mathrm{Y} 1$

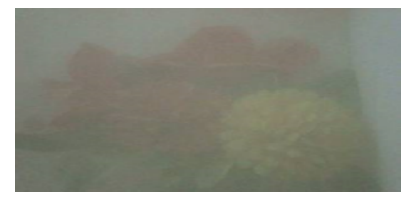

$\mathrm{Y} 2$

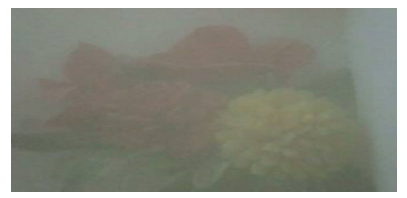

Y3



Y4

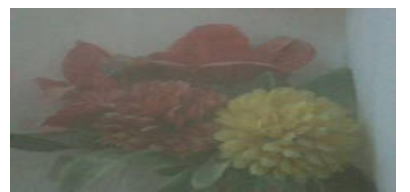

Y5

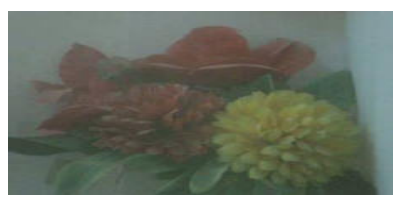

Y6

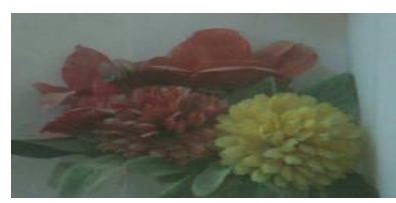

Y7

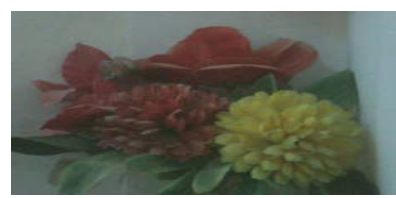

Y8

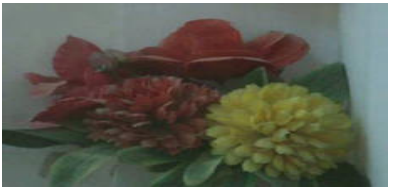

Y9

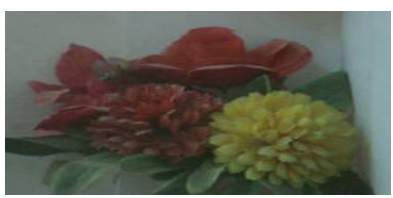

Y10

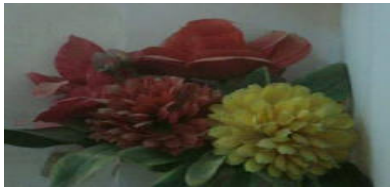

Y11

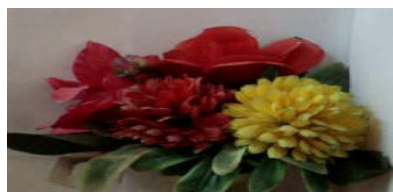

Y12

Figure4. Second group of the dust images with different dust levels frommaximum in y1 to very low in y12



(a)

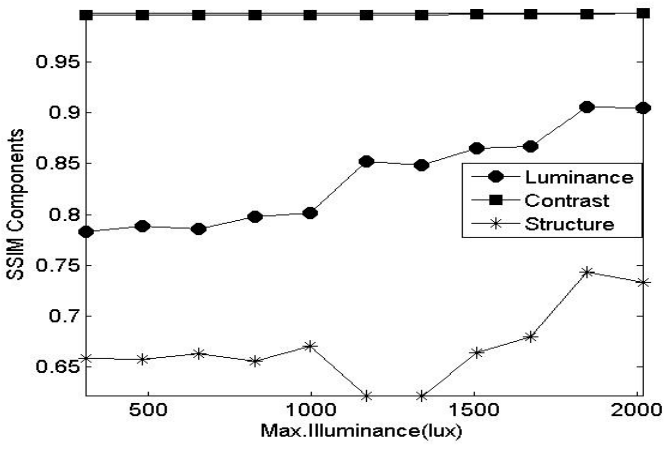

(b)

Figure5. The max.illuminance as a function of SSIM for the value component in YIQ color space (in a) and (in b) the components of SSIM are (luminance, contrast. and structure) for first group images 


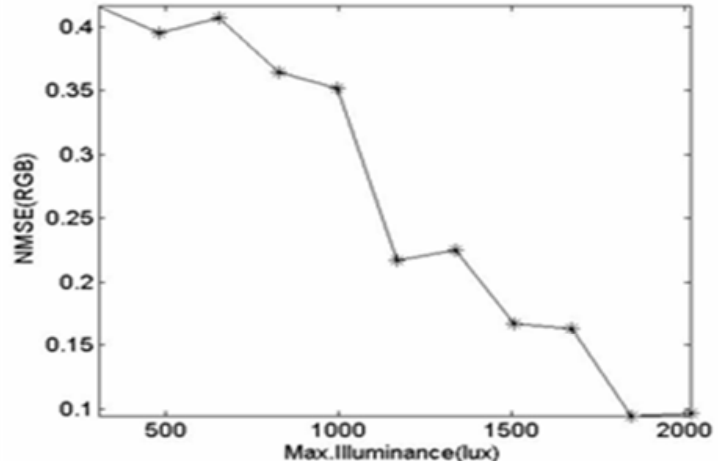

(a)

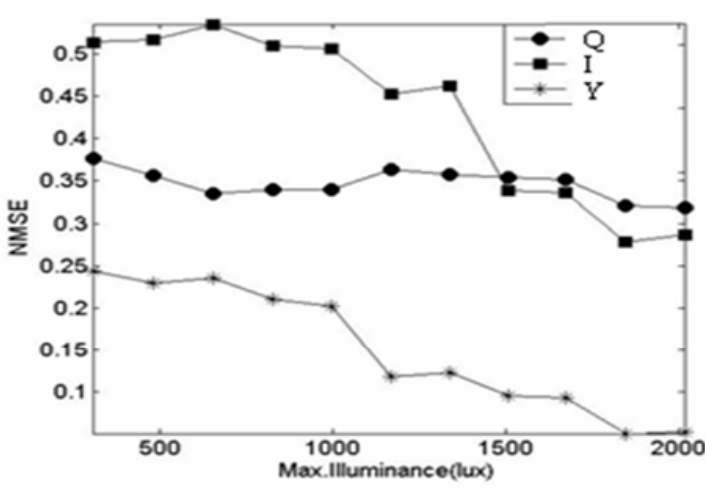

(b)

Figure6. Relationship between Max.illuminance and NMSE (a) for RGB component and (b) for Y,I and Q value components for first group images.

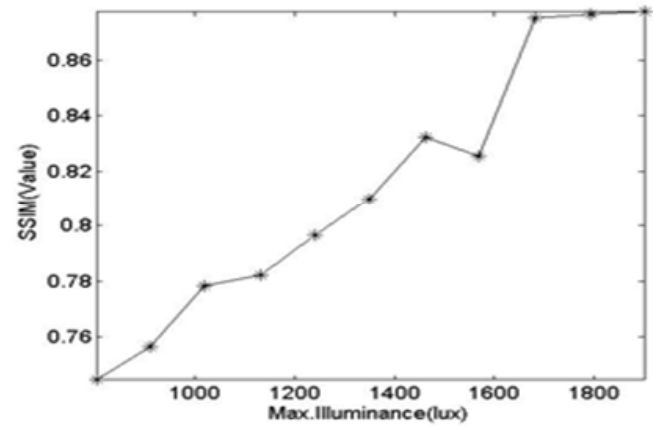

(a)

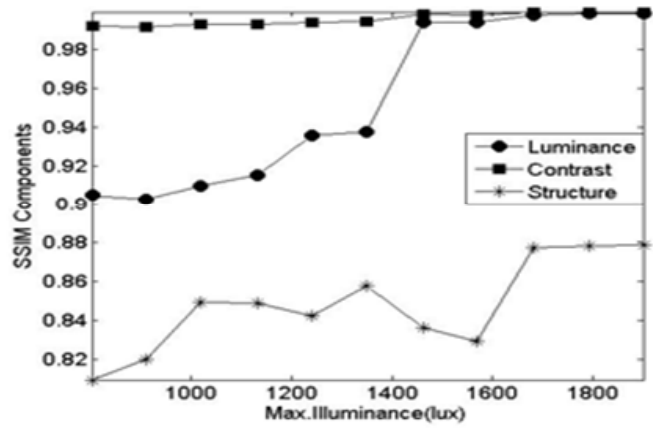

(b)

Figure7. The Max.illuminance as a function of SSIM for the value component in YIQ color space (in a) and (in b) the components of SSIM are (luminance, contrast and structure) for second group images.

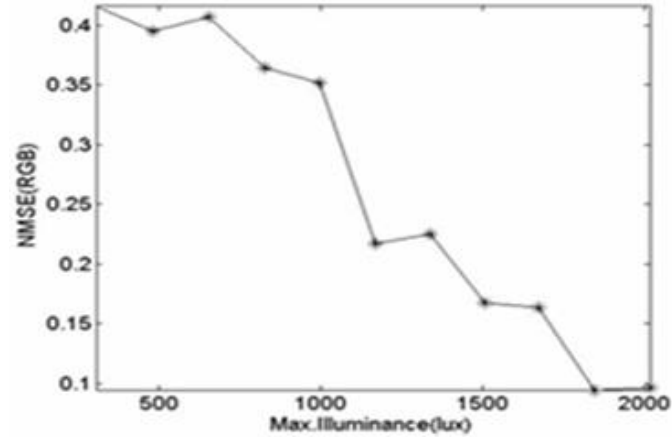

(a)

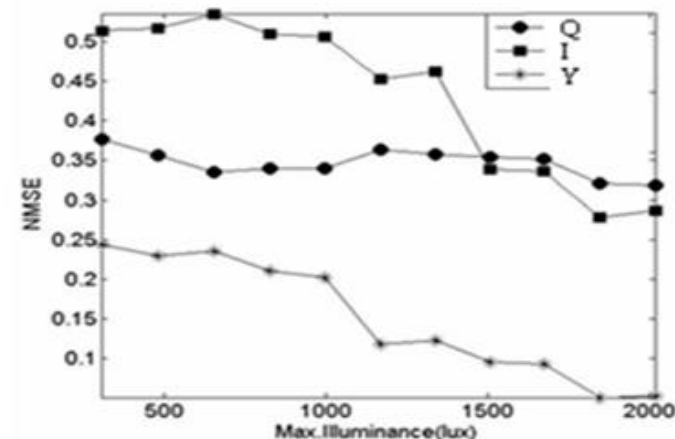

(b)

Figure8. Relationship between Max.illuminance and NMSE (a) for RGB component and (b) for Y,I and Q, value components, for second group images . 


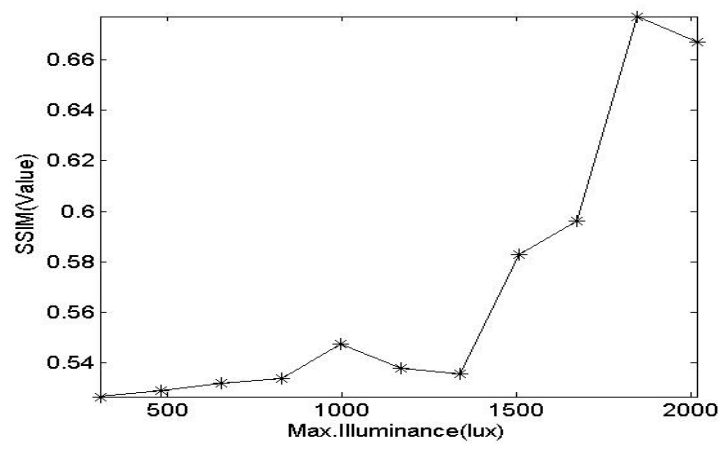

(a)

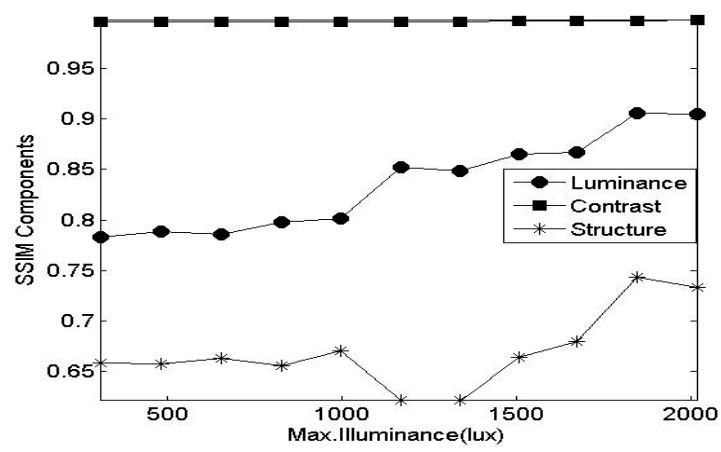

(b)

Figure9. The Max. illuminance as a function of SSIM for the Value component in HSV color space (in a) and (in b) the components of SSIM are (Luminance,Contrast and Structure), for first group images.

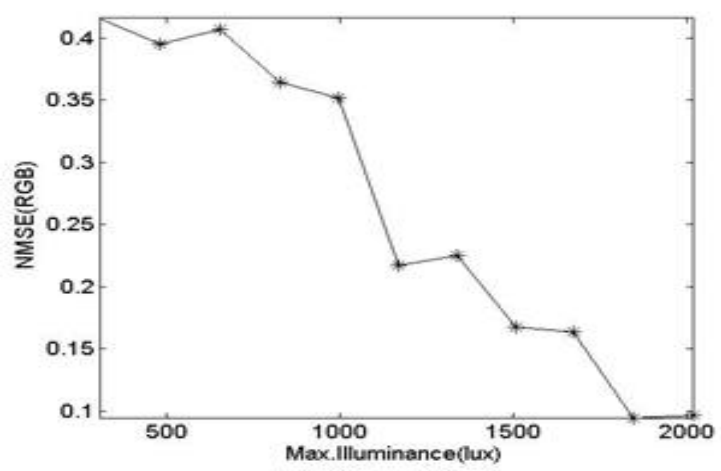

(a)

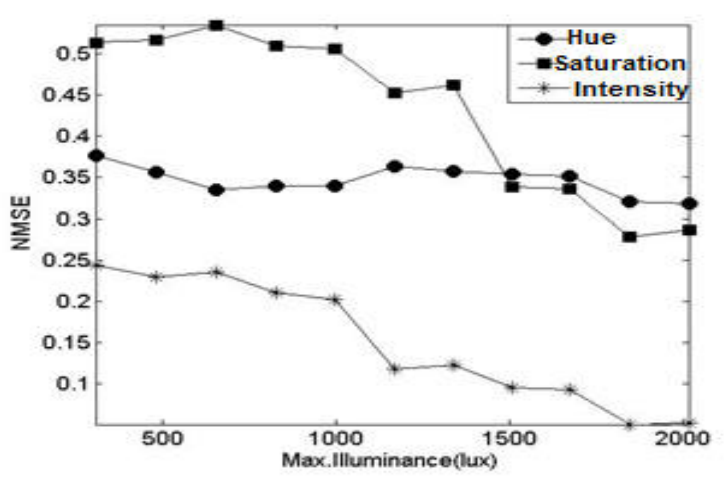

(b)

Figure10. Relationship between Max. illuminance and NMSE (a) for RGB component and (b) for Hue , saturation and intensity value components, for first group images .

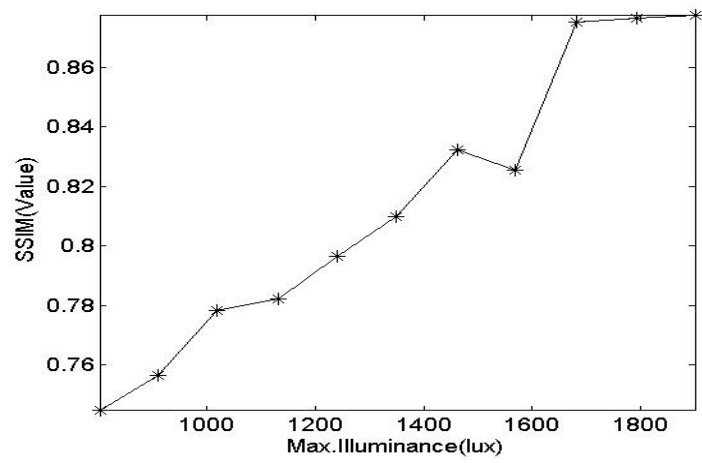

(a)

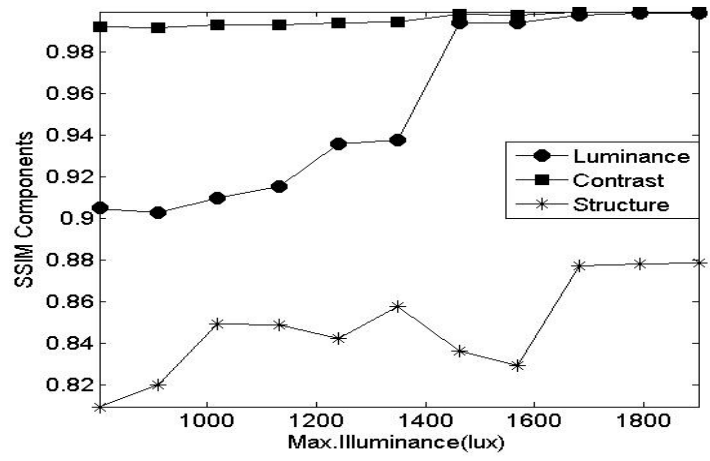

(b)

Figure11. The Max. illuminance as a function of SSIM for the Value component in HSV color space (in a) and (in b) the components of SSIM are (Luminance,Contrast and Structure), for second group images. 




(a)

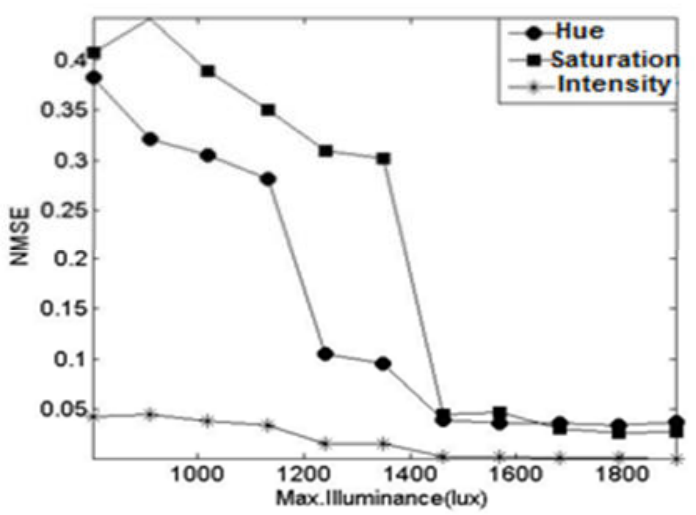

(b)

Figure12. Relationship between Max.illuminance and NMSE (a) for RGB component and (b) for Hue,saturation and intensity value components, for second group images. 